\title{
Mecanismos y factores asociados a la participación parental en la educación en zonas rurales
}

\author{
Forms and Associated factors \\ of parental involvement \\ in rural education
}

$\frac{\text { Ignacio Franco Vega }}{\text { Consultor independiente }}$

Recibido: 1-4-2016

Aprobado: 7-9-2016 


\title{
Resumen
}

La presente investigación tiene como objetivo explorar y describir las formas en las que se manifiesta la participación de los padres en la educación escolar en zonas rurales y los factores que se asocian a ella. A través de entrevistas con familiares de estudiantes, docentes, directores de escuelas y el director de una Unidad de Gestión Educativa Local en la región de La Libertad, se genera un inventario extenso de las diversas formas a través de las cuales los padres participan de la educación de sus hijos, entre las que se encuentran el trabajo en faenas de construcción y en la preparación de desayunos escolares, y el aporte con dinero de forma directa o indirecta, o el apoyo en casa con las tareas de sus hijos. Los principales factores asociados a la participación están relacionados con la búsqueda de mejores condiciones de vida para sus hijos y con el desarrollo de sus habilidades (especialmente, en relación con la lectoescritura) para que puedan migrar a zonas urbanas y continuar su formación, idealmente, siguiendo una carrera profesional. Estos deseos de profesionalización están muchas veces acompañados de una fuerte desvaloración del estilo de vida de los propios padres de familia.

Palabras clave: participación de los padres, relación escuela-comunidad, educación y cultura, educación rural

\begin{abstract}
The aim of this article is to explore and describe how parents get involved in their children's education in rural areas and which factors are linked to this participation. Through interviews with parents, teachers, school principals and the head of one Local Office of Educational Management in the region of La Libertad, I propose an extensive inventory of the different ways in which parents participate, such as the building of the school infrastructure through communal labor, serving breakfast, giving money either directly or indirectly and helping their children with their homework at home. Most of the factors associated with parental involvement are related to the pursuit of better living conditions for their children. Parents support their kids in the development of basic skills (especially literacy abilities) so that they would be able to migrate to urban areas and continue their schooling, ideally towards a professional degree. The desire for their children's professionalization sometimes goes together with a strong sense of loathing and repulsion towards their own life style as farmers.

Keywords: parent participation, school-community relationship, education and culture, rural education
\end{abstract}




\section{Introducción}

De acuerdo con los reportes de la investigación contemporánea, es posible afirmar que la participación o involucramiento de la familia en la escuela tiene un impacto positivo en la calidad del servicio recibido (Cheung \& Pomerantz, 2012; Martini \& Senéchal, 2012; Hill \& Tyson, 2009; McWayne, Hampton, Fantuzzo, Cohen \& Sekino, 2004). Esta relación también se ha observado en contextos latinoamericanos (Murillo, 2007) y peruanos (Cueto, 2007). Sin embargo, el vínculo existente entre resultados educativos positivos e involucramiento parental no siempre es clara. Esto se debe, en parte, a que muchas de las investigaciones realizadas se centran en una sola manifestación conductual del involucramiento parental y conceptualizan esta variable realmente multifacética como un elemento unidimensional (Méndez, 2010; McWayne et al., 2004). Es decir, se generalizan resultados a todo el constructo sobre la base de un número limitado de manifestaciones del mismo (Fan \& Chen, 2001).

La investigación que se desarrolla en el presente artículo tiene como propósito explorar y describir los distintos modos de involucramiento parental y los factores que, según los propios entrevistados, subyacen a esta conducta. El mapeo de forma más detallada de los diferentes mecanismos y factores asociados al involucramiento parental podría facilitar el desarrollo de futuras investigaciones que reconozcan las posibles diferencias generadas por el uso de distintas estrategias, así como la implementación de políticas y programas que incentiven exitosamente el involucramiento de los distintos actores de la comunidad en la labor educativa. El recojo de información se focalizó en zonas rurales de la región de La Libertad. A continuación, antes del análisis de los datos, se presentará una revisión del actual contexto educativo nacional y, luego, una reseña sobre el desarrollo teórico de la variable de involucramiento parental.

\section{Participación parental en el Perú}

En el Perú se han realizado investigaciones que exploraron algunas formas de participación parental en educación (Benavides, Rodrich \& Mena, 2009; Balarin \& Cueto, 2007; Benavides, Olivera \& Mena, 2006). En estas, se identifican una serie de formas frecuentes que serán detalladas. Una primera forma es la participación en la Asociación de Madres y Padres de Familia (Apafa). En esta, se fiscaliza el funcionamiento y gestión de la institución educativa, y se organiza y administra el apoyo financiero y laboral de los padres y madres a la institución. Sobre las Apafa, se han expuesto ciertas preocupaciones. Por ejemplo, Saavedra y Suarez (2002) concluyeron que existía considerable desconfianza hacia las directivas de estas instancias por la percepción de ineficiencia en el manejo del dinero, a pesar de que eran elegidas por los propios padres. Benavides et al. (2009) sostienen que la participación en la Apafa no se correlaciona con actividades de acompañamiento escolar en el hogar. Por otro lado, 
la capacidad de la Apafa para participar en la gestión de la IE ha sido relativamente limitada. Gonzales (2006) sostiene que ha existido una división tácita entre las competencias de las Apafa (asistencia financiera y laboral), y las de los directores y docentes (gestión pedagógica e institucional). Esta separación se ha mantenido en casi todas las ocasiones, a pesar de que el marco normativo que regulaba su accionar ha ido ganando y perdiendo ámbitos (legales) de participación en distintos momentos.

La segunda forma de participación consiste en la realización de asambleas, faenas y otros trabajos comunales para la escuela, especialmente, relacionados con el financiamiento de materiales educativos y equipamiento, así como con la construcción de infraestructura. Millán y Lossio (2005), y Montero (2006) resaltan la importancia histórica de este modo de involucramiento en la política nacional. Finalmente, la tercera forma refiere a la vigilancia del cumplimiento de tareas escolares y la asistencia al centro de estudios. Esta se da en menor proporción que las dos anteriores y con gran variabilidad entre los padres.

Como un elemento adicional, se incorpora a esta lista el espacio de participación propuesto por el Estado mediante la Ley General de Educación (Ley No 28044, 2003): los Consejos Educativos Institucionales (Conei). Estos consejos son precedidos por el director de la IE con la participación de algunos padres de familia, docentes, alumnos y exalumnos. Han sido planteados con miras a mejorar la eficiencia de la institución educativa, tanto en aspectos pedagógicos como de gestión y transparencia (Balarin \& Cueto, 2007). Benavides et al. (2006) consideran que la participación dentro de los Conei es una réplica de la ya existente desvinculación de los temas familiares-escolares, solo que en un nuevo espacio, y que podría conducir a un aumento de las brechas educativas entre las áreas urbanas y rurales. Esto se debe a que las políticas de participación y democratización se ejecutan en mejor y mayor medida en escuelas polidocentes, más grandes y urbanas, asociadas a familias hispanohablantes y de mayor nivel socioeconómico (Benavides, 2007). Debe recordarse que las escuelas de zonas rurales manejan un nivel de autonomía elevado, a razón de las distancias que las separan de las instancias descentralizadas de gestión. Esta libertad genera condiciones muy diferentes en cada escuela, situación que se refleja también en la participación de las familias (Ames, Rojas \& Portugal, 2009). Para cambiar esta situación, Eguren (2006) sugiere el diseño e implementación de programas para ámbitos específicos que permitan corregir las inequidades mediante intervenciones diferenciadas. Esta misma autora enfatiza que el problema radica en la sostenibilidad de las iniciativas y que los mayores logros en la participación se dan cuando esta se promueve desde los niveles inferiores del sistema, no cuando es dictada desde arriba.

En general, se encuentra que la concepción actual de participación parental está vinculada a proporcionar las condiciones materiales mínimas para el funcionamiento de la escuela y, en menor medida, a fiscalizar el cumplimiento de las tareas (Benavides et al., 2009). Según Saavedra y Suarez (2002), el pro74 I pio sistema de educación pública requiere, para su funcionamiento, el aporte 
económico de las familias, con lo que se logra cubrir directamente un tercio de la inversión total de cada niño.En lo concerniente a los factores asociados a la participación de los padres, Benavides et al. (2009) identificaron que los principales motivos para la participación en actividades escolares eran extrínsecos: existe una considerable preocupación de los padres por evitar la multa que conlleva no asistir a las asambleas. Además, se notó que no todos los asistentes participaban efectivamente, sino que asumían una presencia pasiva.

\section{Marco conceptual}

Una vez establecido el contexto en el que se realizó el estudio, se realizará la revisión de la variable: ¿Qué es exactamente participación o involucramiento parental? Pese a que la investigación sobre este concepto se ha desarrollado ampliamente, subsisten en ella algunas dificultades teóricas y, específicamente, una limitada concertación de conceptos (Kyriakides, 2005). En diversas investigaciones se han priorizado distintas conductas específicas (como acompañamiento al desarrollo de tareas en casa o lectura de cuentos antes de dormir) que, luego, han sido generalizadas bajo el concepto general de involucramiento parental. La amplitud de conductas categorizadas como manifestaciones del concepto contribuyen a la inconsistencia de hallazgos de las distintas investigaciones sobre los efectos particulares del involucramiento en los diversos resultados educativos (Fan \& Chen, 2001).

Dados los objetivos exploratorios de la presente investigación, se ha optado por una definición abierta: se considera que el involucramiento parental consiste en la posibilidad de incidir, decidir, aportar y disentir en diversos campos de la educación, acordados previamente entre docentes, padres y otros agentes educativos, con funciones definidas y comprendidas por ambos agentes (Orealc/Unesco, 2004).

Uno de los principales esfuerzos de sistematización es el Hoover-Dempsey y Sandler (1997), quienes presentan un modelo de cinco niveles que articula diversas variables relacionadas con las causas y consecuencias del involucramiento parental. Para este estudio, se trabajará con un subcircuito del modelo; solo se revisarán los dos primeros niveles de la sistematización: los factores asociados al involucramiento y las formas en las que este se concreta. 
Gráfico 1. Cinco niveles del modelo de participación parental de Hoover-Dempsey y Sandler ${ }^{1}$

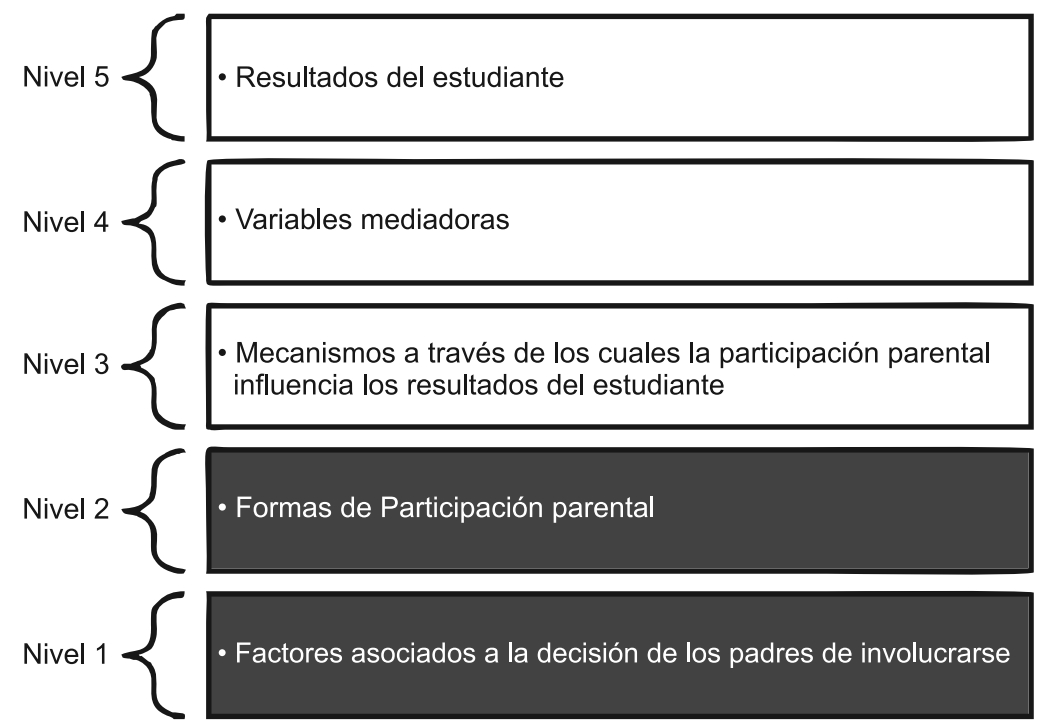

Fuente: Walker, Wilkins, Dallaire, Sandler \& Hoover-Dempsey (2005) Elaboración propia

Los autores exponen tres grandes componentes vinculados a la participación y, dentro de cada uno de estos, se presentan dos o tres subelementos que definen más claramente la dinámica de cada componente. Las formas de involucramiento se dividen en dos grupos: el primero de ellos se realiza a través de la participación en el hogar, es decir, el seguimiento a las tareas, las conversaciones sobre la situación actual del colegio, etc. El segundo está orientado a la escuela; en otras palabras, considera la intervención que puede realizar la familia en temas pedagógicos y de gestión institucional dentro de los centros educativos (Christenson \& Sheridan, 2001).

A continuación, se presenta un breve resumen del modelo, así como algunos hallazgos adicionales y la descripción de cada elemento en el contexto local.

1. Se resaltan los niveles tomados en cuenta para este documento. 
Gráfico 2. Los primeros dos niveles del modelo de Hoover-Dempsey y Sandler (2005)

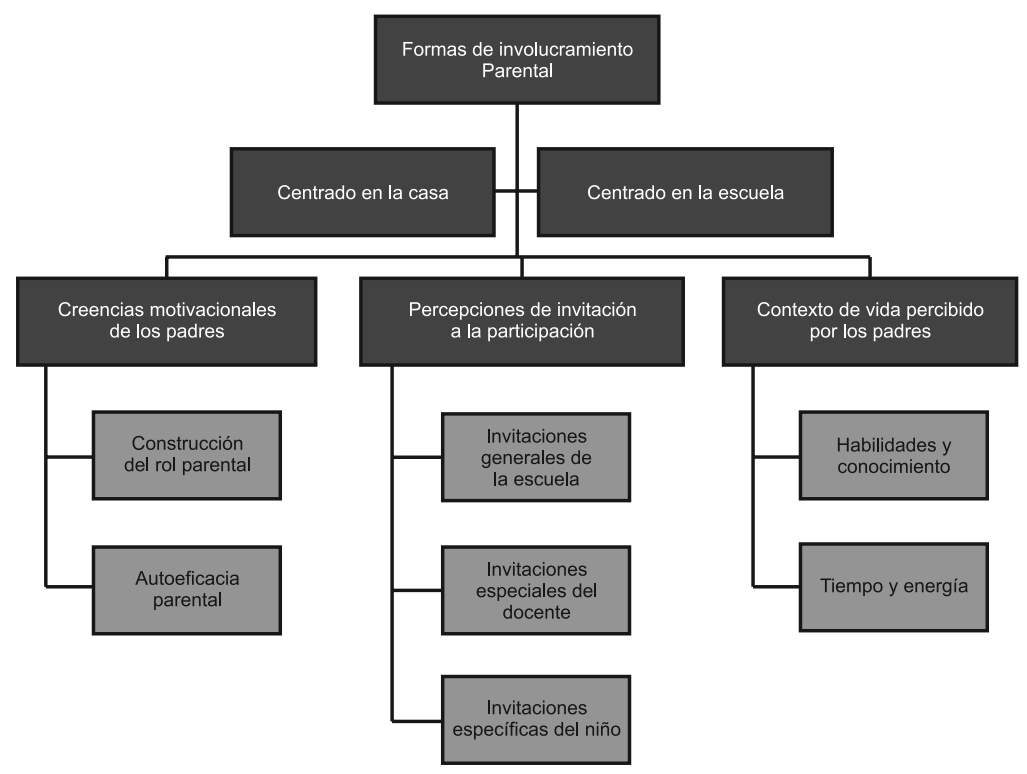

Fuente: Walker et al. (2005)

Elaboración propia

\subsection{Creencias motivacionales de los padres}

La construcción del rol parental es un proceso social en el que toman parte las propias experiencias del sujeto, así como los modos de conducta que ha aprendido y las expectativas que la sociedad tiene de él. Este tema se relaciona con los patrones de crianza propuestos por Lareau (2002), quien conceptualiza dos extremos en un continuo de involucramiento en la crianza. En un extremo, se encuentra el crecimiento natural, característico de familias de bajos ingresos. En este, se considera que el niño debe desarrollarse de forma libre y natural, obedeciendo siempre las direcciones de sus padres o cuidadores; los niños mantienen un grupo de pares de edades heterogéneas y fuertes vínculos con la familia ampliada. En el otro extremo, el otro patrón de crianza es el del cultivo concertado. En este, el padre organiza los espacios y las actividades en las que el niño se desempeña para favorecer un objetivo en particular. También, se hace hincapié en el tema de la concertación entre el niño y los cuidadores. Estos últimos tienen derecho a realizar críticas si es que no están de acuerdo con un modo de accionar o una decisión en particular.

Es importante recalcar que estos modos de crianza son socialmente heredados. Se ha encontrado que padres que poseen un rol más activo (entiéndase cultivo concertado) tienden a involucrarse más en la educación de los 
hijos (Deslandes \& Bertrand, 2005). La estructura y dinámica de las familias de las zonas rurales descritas por Bello y Villarán (2004) incorporan muchas características del extremo de crecimiento natural. En la zona rural, al evaluar el involucramiento parental, Balarin y Cueto (2007) concluyeron que pocos padres, especialmente aquellos con hijos con bajo rendimiento académico, podían especificar su rol en relación con el proceso de aprendizaje del niño. Asimismo, Ames et al. (2009) constatan que los padres consideran elementos bastante generales en su concepción de apoyo a la educación. Acciones como comprarles útiles, mandarlos a la escuela y alimentarlos bien son percibidas como sus principales obligaciones. Estas creencias no deben ser confundidas con desinterés por la participación necesariamente, sino entendidas como una construcción sobre el rol que debe desempeñarse. Al respecto, Bello y Villarán (2004) señalan que, a mayor experiencia en el sistema educativo de los padres, mayor será la diversidad de las conductas de involucramiento que puedan ejecutar.

Dentro de este componente, se incluyen las aspiraciones de los padres. Se ha encontrado evidencia, tanto a nivel internacional (Fan \& Chen, 2001) como local (Balarin \& Cueto, 2007; Cueto, 2004), de la enorme importancia de las aspiraciones de los padres en la educación de los hijos. Carranza, You, Chhuon y Hudley (2009) especifican que no son las aspiraciones de los padres, sino, más bien, la percepción que los niños tienen de estas las que se relacionan con el logro académico. Este aspecto ha sido ampliamente estudiado en el ambiente local: la profunda valoración que se le da a la instrucción se da con la esperanza de lograr un ascenso social. Este llamado «Mito de la educación» (Ames, 2002; Ansión, 1995) se encuentra vinculado con los procesos migratorios y un acercamiento, en caso de poblaciones hablantes de quechua o aimara, a la lengua castellana (Bello \& Villarán, 2004). En este marco, se considera que la educación abre las puertas para la adquisición de un estatus social superior mediante el acceso a nuevas oportunidades de desarrollo en las ciudades.

En lo concerniente a la autoeficacia parental, diversos estudios han observado que aquellos padres que creen que su intervención tendrá un efecto beneficioso tenderán a involucrarse más (Hoover-Dempsey \& Sanders, 1997; Hoover-Dempsey, Battiato, Walker, Reed, DeJong \& Jones, 2001). Los autores presentan como hipótesis que los padres que se involucran en la educación normalmente obtienen algún nivel de resultados positivos y tienen la tendencia de atribuir estos, en parte por lo menos, a su propio esfuerzo y al del niño. La consecuencia de este proceso refuerza la conducta de involucramiento.

Teniendo como soporte está orientación motivacional, hay tres factores que pueden conducir a la ausencia de involucramiento parental. La primera es que no se percibe necesario involucrarse, puesto que el estudiante ya se está desempeñando bien (Méndez, 2010). La segunda razón que justifica la ausencia del involucramiento es la desconfianza en las propias habilidades para el área en general. La última opción es que los padres o cuidadores no consi78 I deran que el modo en el que se enseña ahora es compatible con el que les fue 
brindado a ellos (Gu, 2008; Balarin \& Cueto, 2007; Benavides, 2007; Benavides et al., 2006).

\subsection{Percepción de invitación para el involucramiento parental en educación}

En lo concerniente a las invitaciones generales de la escuela, Lareau (2002) postula que el sistema educativo es el principal moldeador de los espacios de participación. Se considera que son la escuela y los ámbitos jerárquicamente superiores a esta los que orquestan, facilitan o dificultan la participación familiar. El modelo propone también la importancia de las invitaciones específicas del docente, especialmente, de la percepción de los padres sobre las mismas (Hoover-Dempsey et al., 2001). Se ha reportado que existen ciertos elementos que inhiben la invitación de los docentes: existe incomodidad ante la presencia de agentes extraños (las familias) en los ambientes escolares, sobre todo, si es que estos van a ser capaces de ejercer algún tipo de decisión (Hung, 2007; Eguren, 2006). Los docentes, a veces, no consideran dicha presencia necesaria, porque creen que los padres no serán capaces de ejercer algún efecto beneficioso. En algunos casos, incluso, se percibe que la cultura y entorno social del educando son los causantes del bajo desempeño (Ames et al., 2009). Finalmente, no se involucra a las familias por temor a no poder responder adecuadamente a las expectativas de los padres (Gu, 2008; Orealc/Unesco, 2004).

\subsection{Contexto de vida percibido por la familia}

Las habilidades para el involucramiento de los padres se relacionan con su autoeficacia e incluyen el manejo específico de contenidos educativos para una exitosa participación (operaciones matemáticas, historia universal, castellano, etc). Se ha encontrado que, en el contexto local, ante la falta de conocimiento de los padres, muchas veces son los hermanos mayores los que asumen el rol de apoyo en las tareas escolares (Benavides et al., 2009). Estas dificultades en el manejo de conocimientos específicos se incrementan cuando los niños realizan la transición de primaria a secundaria, dado que las instituciones educativas dedicadas a este nivel tienden a ser más grandes y burocráticas $(\mathrm{Gu}, 2008)$.

Emanique y Earl (2009) plantearon que el nivel educativo de los padres y la presencia de capital cultural en casa están asociados con un mayor involucramiento en la escuela. En zonas rurales del Perú, se han reportado resultados similares: un mayor nivel educativo de los padres y madres se vinculaba con una mayor dedicación de tiempo al desarrollo de tareas escolares (Benavides et al., 2009), así como a conductas generales de involucramiento (Bello \& Villarán, 2004). Sin embargo, Kyriakides (2005) encontró que, mediante una guía adecuada, los padres eran capaces de apoyar a sus hijos incluso en temas en los que ellos inicialmente no se sentían competentes. Ahora bien, en el contexto local, no se evidencia esta guía (Balarin \& Cueto, 2007; Benavides, 2007), ya sea porque los profesores no la otorgan o porque 
las familias no la buscan. Al respecto, Yan y Lin (2005) encontraron que, ante la percepción de que los valores familiares-culturales no se transmiten en la escuela, las familias optaban por no involucrarse. Estos autores hallaron barreras en lo que respecta al propio idioma y revelaron que existían familias que preferían no involucrarse, puesto que no compartían la misma lengua que la utilizada en la escuela. Estas condiciones son similares a las encontradas en la sierra y selva peruanas (Balarin \& Cueto, 2007). En relación con ello, algunos autores sostienen que existe incluso un desacoplamiento entre las características del ambiente rural y las formas de aprendizaje escolar (Belaunde, 2006), lo cual ocurre debido a las diferencias en los mecanismos de aprendizaje propios del ambiente rural (principalmente, observacional-empírico) y los modos propuestos por el sistema educativo (de carácter más abstracto y centrado en modos más urbanos de pensamiento).

Se propone que, dada la multiplicidad de conductas que se incluyen en las diversas definiciones de la variable involucramiento, este debe ser considerado como un elemento multifacético más que unidimensional (Méndez, 2010; Fantuzzo, Bulotsky-Shearer, McDermott, McWayne, Frye \& Perlman, 2007; McWayne et al., 2004; Fantuzzo, McWayne, Perry \& Childs, 2004; Fan \& Chen, 2001; Christenson \& Sheridan, 2001). La conceptualización del involucramiento como un constructo complejo podría facilitar la interpretación de las distintas evaluaciones sobre la relación entre participación y otros constructos, en la medida que permitiría diferenciar los efectos de los distintos modos de involucramiento de forma independiente, en lugar de tomarlas como una sola variable. Esto también favorecería el desarrollo de estrategias de intervención más efectivas, en tanto podrían focalizarse en los elementos del involucramiento más eficientes para el logro de los objetivos particulares.

El modelo presentado por Hoover-Dempsey y Sandler (1997) facilita la organización de los distintos estudios que se han realizado sobre la participación familiar en la educación. Como se ha observado, son múltiples los factores que influyen en el involucramiento y múltiples, además, las manifestaciones de este. Ante la amplia gama de elementos que influyen en esta variable y la importancia del contexto en el que esta se da (Carranza et al., 2009), es crucial el desarrollo de un estudio que explore estos elementos en el ambiente rural, dadas las particularidades que este presenta.

\section{Método}

A través de la presente investigación, se buscó realizar una aproximación inicial al fenómeno del involucramiento familiar. Se tuvo como objetivo recoger información de las formas y factores asociados a la participación en educación. A partir de este conocimiento, se crearon categorías para un análisis más esquemático, que permitió aislar las unidades básicas con las que funciona el constructo y las peculiaridades propias del contexto. 


\subsection{Participantes}

La muestra estuvo constituida por diferentes actores educativos en el ámbito de la Ugel Julcán en la región de la Libertad. Se trabajó con familiares de los estudiantes (37 en total: 1 abuelo, 1 hermano, 20 madres y 15 padres), directores y docentes ( 8 entrevistas, de las cuales 2 fueron grupales), así como con el director de gestión pedagógica e institucional de la UGEL Julcán ${ }^{2}$. En total, se manejaron 46 entrevistas. Se optó por trabajar con distintos actores de la comunidad educativa para comprehender la mayor diversidad posible de perspectivas respecto a las variables estudiadas.

Se trabajó en seis escuelas/comunidades y en dos distritos de la provincia. El muestreo fue no probabilístico intencional, tanto a nivel de las escuelas (donde se eligió una escuela unidocente, una multigrado y una polidocente por distrito), como de los familiares, pues se entrevistó a aquellos dispuestos a responder las preguntas. Se trabajó con este sistema, porque se buscaba incorporar la diversidad de tamaños de escuelas bajo el supuesto de que esto podría tener un efecto en las variables a evaluar.

\subsection{Medición}

Para el recojo de información, se utilizó una entrevista estructurada con preguntas abiertas de elaboración propia, que tenía como objetivo recoger información sobre los modos y factores asociados a la participación familiar en la educación en esas comunidades. El diseño se realizó sobre la base de la revisión de estudios anteriores con temáticas y población similar. Luego de la elaboración inicial, la guía fue revisada por personal de la Ugel Julcán y piloteada en la zona. Tras esta aplicación inicial, la guía fue modificada para adecuarse de mejor manera al lenguaje y particularidades de la localidad. Se decidió optar por una versión compuesta para la pregunta sobre modos de participación para asegurar que se incorporen tanto aquellos relacionados con el trabajo en la institución como los propios de la labor en casa.

\subsection{Procedimiento}

Se realizó un acercamiento a la Ugel Julcán para negociar la posibilidad de la investigación. Una vez establecidos las concesiones y los acuerdos, se seleccionó a las escuelas. Ya en la comunidad, se realizó una presentación inicial al director para, luego, proceder de la misma manera con los docentes y los padres de familia que se encontraban en la zona.

La validez de la guía se aseguró de tres formas. La primera fue a partir de la revisión de la guía por personal de la Ugel con experiencia en la zona; la segunda, a través del piloto en una población similar; y, finalmente, a partir de la

2. Por el tamaño de la Ugel, una misma persona está a cargo de ambas direcciones. 
revisión de las respuestas con los entrevistados durante la aplicación. Mediante estos tres procesos, se aseguró tanto la comprensión de las preguntas como la fiabilidad de las respuestas.

\subsection{Análisis de datos}

Se analizó el contenido de cada una de las variables evaluadas. Para garantizar la confiabilidad de la categorización, se solicitó a una investigadora especialista en educación rural que revise las etiquetas de las categorías y que, basándose en estas, realice un proceso de categorización independiente. Se obtuvo una consistencia entre ambos evaluadores de $93,71 \%$ en «Modos» y $88,59 \%$ en «Factores asociados». Producto de la revisión de esta especialista se definieron las etiquetas de tres categorías.

\section{Resultados}

A continuación, se presentan la tabla en las que se resume la frecuencia con la que los distintos modos de involucramiento identificados se hallan en las entrevistas:

Tabla 1. Listado de mecanismos de involucramiento parental

\begin{tabular}{clc}
\hline$\#$ & \multicolumn{1}{c}{ Modos de involucramiento } & f \\
\hline Mo01 & Realizar trabajo comunal para construir/mejorar la infraestructura de & 30 \\
Mo02 & Apoyar en la realización de tareas en casa & 19 \\
Mo03 & Cumplir con reuniones y pagos de la Apafa & 13 \\
Mo04 & Dar apoyo material/financiero al colegio & 11 \\
Mo05 & Asegurar la asistencia y puntualidad del docente & 10 \\
Mo06 & Revisar cuadernos y exigir tareas y estudio & 9 \\
Mo07 & Participar en actividades culturales y profondos & 9 \\
Mo08 & Reunirse/conversar directamente con el docente & 8 \\
Mo09 & Preparar desayuno/almuerzo en el colegio & 8 \\
Mo10 & Motivar y aconsejar a sus hijos & 7 \\
Mo11 & Enviar/matricular a sus hijos & 6 \\
Mo12 & Dar buena alimentación en casa & 5 \\
Mo13 & Complementar la educación de la escuela con lecciones de los propios & 5 \\
podr & Participar del programa Juntos & 4 \\
Mo15 & Reunirse/conversar directamente con director & 3 \\
Mo16 & Mejorar el aseo del niño & 3 \\
Mo17 & Trabajar más para que su hijo no tenga que hacerlo & 3 \\
Mo18 & Asegurar la calidad de la enseñanza del docente & 3 \\
\hline
\end{tabular}

Fuente: Elaboración propia 
Sobre la base de lo precisado por los entrevistados, se determinaron dieciocho formas de participación en la educación escolar. Como puede observarse, hay dos claramente dominantes: el trabajo comunal en la infraestructura de la IE y el apoyo en la realización de las tareas en casa. El primero (Mo01) consiste en el cumplimiento de las cuotas de trabajo físico en construcción y mantenimiento del local escolar. Estos trabajos son organizados, en la mayoría de casos, por la Apafa en coordinación con la dirección de la IE y los acuerdos se manifiestan, a su vez, en el Plan Anual de Trabajo de la Asociación. En casi la totalidad de los casos, esta actividad es realizada por el padre de familia con materiales comprados con el dinero de la Apafa, donados por el Gobierno local, por los propios padres de familia o adquiridos a través de gasto ordinario del Ministerio para el mantenimiento de las aulas. Existe un sistema similar en el modo «Preparar desayuno/almuerzo en el colegio» (Mo09), que está organizado en turnos, y es realizado exclusivamente por las madres con insumos brindados por las propias familias o por el gobierno.

El trabajo en casa se relaciona con las tareas escolares y el estudio. En el primer caso, el apoyo en la realización de tareas en casa (Mo02) consiste en la asistencia al momento de hacer las labores escolares en la vivienda. Otro elemento relacionado es la revisión y exigencia de tareas (Mo06), muy vinculada con el referente concreto del avance en el cuaderno; sin embargo, hay un matiz diferente, en tanto en este el foco está en la vigilancia al cumplimiento más que en la asistencia al mismo. Estas formas de participación no son necesariamente excluyentes. En la casa, también, se dan otras actividades que son adicionales al cumplimiento de las tareas. Existe otra categoría en la que los propios familiares desarrollan «lecciones» que explican a los hijos (Mo13). Los primeros también motivan y aconsejan a los alumnos para que se mantengan en la escuela, y los animan a que se esfuercen en la misma (Mo10). De forma menos frecuente, también, se encuentran acciones como la mejora de la alimentación (Mo12) y el aseo de los hijos (Mo16).

En lo concerniente a la Apafa (Mo03), hay dos aspectos importantes. El primero consiste en la asistencia a las reuniones. Estas pueden ser por secciones, promociones o de todo el colegio, dependiendo del tema a tratar o del tamaño de la institución. Los temas a tratar en estas reuniones suelen estar vinculados a las mejoras de la infraestructura del local, o la adquisición de bienes para equipamiento de la IE o útiles extra para los alumnos. El segundo elemento es el pago de cuotas de Apafa. Estas últimas tenían la característica de ser bastante flexibles: a veces, anuales o semestrales. Además, las familias de la muestra también participan mediante concursos y eventos culturales (Mo07). Existe una amplia gama de actividades en este rubro, desde competencias deportivas a concursos de platos típicos y verbenas. En algunos casos, estas se realizan con el objetivo de financiar la compra de materiales de construcción o de útiles, pero también pueden realizarse con fines de integración.

Se encontraron otros elementos relacionados al personal de la propia institución. Por ejemplo, se llevan a cabo reuniones directas con el docente (Mo08) 
y con el director (Mo15); estas últimas son menos frecuentes que las primeras (aunque esta diferenciación podría verse afectada por la doble función del docente/director en colegios unidocentes). En estas reuniones, se discute sobre el avance de los estudiantes y, especialmente, se informa de aquellos cursos en los que no se está desempeñando satisfactoriamente. También, se justifican las faltas de sus hijos (esto es especialmente importante debido a las condiciones para obtener el bono del programa Juntos) y se confronta al docente en caso no esté realizando su trabajo. Los familiares, también, fiscalizan el trabajo de este. Con mayor frecuencia se controla la asistencia y puntualidad (Mo05), en lugar de aquellos temas vinculados con la calidad en los procesos de enseñanza (Mo18).

Adicionalmente, existen dos elementos particulares de la muestra que son dignos de mención: la consideración de que enviar y matricular a los hijos es, en sí misma, una forma de participación (Mo11), y el trabajar más para que los hijos no tengan que hacerlo (Mo17) y puedan dedicarse casi en totalidad a sus estudios. A continuación, se presenta la tabla de frecuencias que describe lo obtenido respecto a los factores percibidos como asociados al involucramiento:

Tabla 2. Listado de factores asociados al involucramiento parental

\begin{tabular}{clc}
\hline$\#$ & \multicolumn{1}{c}{ Factores asociados al involucramiento } & $\mathrm{f}$ \\
\hline Fc01 & Buscan que sus hijos sean profesionales. & 24 \\
Fc02 & Buscan que sus hijos no sean como ellos. & 18 \\
Fc03 & Buscan que sus hijos se superen. & 13 \\
Fc04 & Desconocen los contenidos escolares. & 10 \\
Fc05 & Están obligados. & 9 \\
Fc06 & Es su responsabilidad como padres. & 7 \\
Fc07 & Buscan mejorar la calidad del aprendizaje de sus hijos. \\
Fc08 & $\begin{array}{l}\text { Buscan que sus hijos sean fuente de orgullo y desarrollo de la familia y } \\
\text { comunidad. }\end{array}$ & 7 \\
Fc09 & Los cambios en la educación no se lo permiten. & 6 \\
Fc10 & Actúan de acuerdo con lo que los demás piensen. & 5 \\
Fc11 & $\begin{array}{l}\text { Buscan mejorar las condiciones físicas, de seguridad y estéticas de la } \\
\text { infraestructura de la IE. }\end{array}$ & 4 \\
\hline
\end{tabular}

Fuente: Elaboración propia

Se observa la presencia de once factores. Dos de estos se muestran dominantes frente al resto. El primero es «Que no sean como ellos» (Fc02); en este caso, se presentan juicios negativos hacia el propio modo de vida, cultura y actividad económica de los familiares. Esta categoría se diferencia de «Buscan 84 I que sus hijos se superen» (Fc03), debido a que esta última no presenta un tinte 
de desvalorización a la situación actual tan evidente como la primera. La razón más recurrente está relacionada también con las dos anteriores, pues, en esta categoría, se incorporan todas las aseveraciones asociadas a la profesionalización de los hijos ( $\mathrm{F} 01)$. Se encuentran algunas razones que subyacen a la profesionalización de los hijos. La primera es que esto les facilitará un buen trabajo más adelante; la segunda es para que sean independientes y, en caso sea necesario, retribuyan la inversión de tiempo y dinero.

Existe otra categoría, normalmente explicitada por los docentes, que sostiene que los familiares participan por obligación (Fc05). Este conjunto incorpora tanto la obligación que genera el profesor como la presión de las multas de Apafa y la condicionalidad del bono brindado por el programa Juntos a la asistencia al colegio.

Por su parte, algunos entrevistados consideraban que era necesario participar, dado que existían ciertas carencias en el sistema educativo que hacían imperativo su involucramiento para un aprendizaje adecuado. De forma similar, se encontró que, en algunos casos, se buscaba mejorar la calidad ( $\mathrm{Fc07}$ ) no necesariamente porque estaba mal, sino porque podía estar mejor. En otros casos, se evidenció que el motivo es que el involucramiento es una responsabilidad, entiéndase deber, de los padres ( $\mathrm{Fc} 06)$.

En una categoría, se incorpora la posibilidad de que los hijos, con su aprendizaje mejorado, logren fomentar el desarrollo de la propia comunidad convirtiéndose en fuente de orgullo para la familia (Fc08). Finalmente, el desconocimiento de los contenidos escolares ( $\mathrm{Fc} 04)$ y el efecto de los cambios en la educación ( $\mathrm{Fc09}$ ) son categorías particulares por su carácter inverso. Es decir, explican por qué los padres no se involucran en la educación de los hijos.

Una vez realizada la categorización y descripción de las variables, estas fueron organizadas sobre la base del modelo teórico. A continuación, se presenta una réplica del esquema utilizado en el marco conceptual adicionando las categorías creadas durante el estudio. En la mayoría de los casos, las categorías podían adscribirse a un elemento particular del modelo, pero, en dos ocasiones, en las que los hallazgos no se adecuaban a la estructura predefinida, se consideró pertinente la creación de elementos adicionales para registrar las particularidades del contexto investigados. 
Gráfico 3. Modos y factores asociados a la participación, encontrados durante la investigación y organizados alrededor del modelo teórico

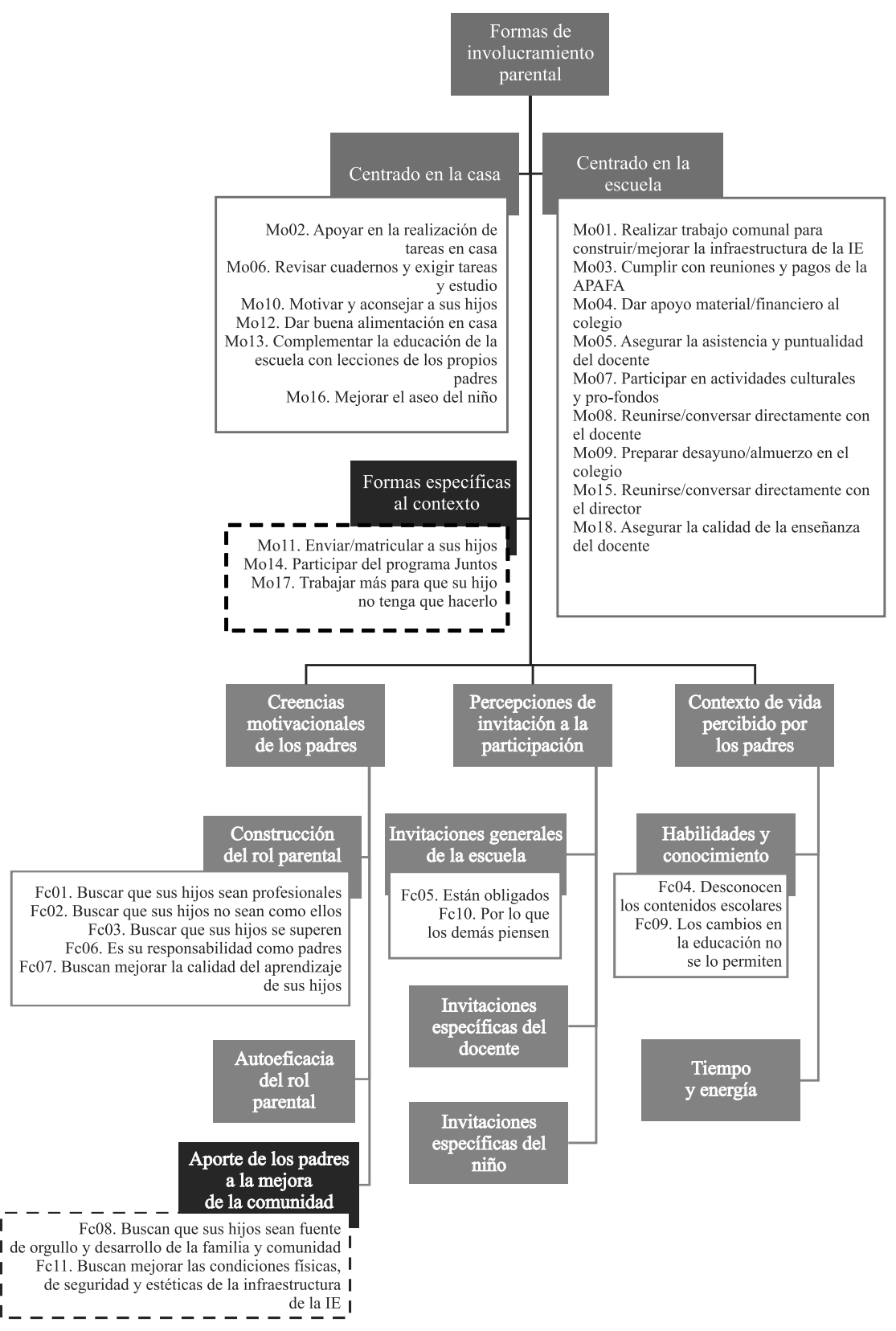




\section{Discusión}

La discusión de los hallazgos se ha organizado sobre la base de los puntos descritos en el marco conceptual. A continuación, se desarrolla cada uno de los mismos.

\subsection{Modos de participación}

En lo que concierne a los modos en lo que los padres se involucran en la educación de los hijos, encontramos algunos puntos destacables. Tanto los modos centrados en la casa como en la escuela son implementados. En lo concerniente a la participación en la institución, puede observarse que existe una considerable presencia de mecanismos de participación a través de la provisión de servicios para la escuela (limpieza, construcción, cocina, etc.). Estas estrategias de participación son coherentes con las formas de trabajo comunitario tradicional de las zonas rurales (Ansión, 1995). Por otro lado, son el único modo de involucramiento registrado que ha presentado diferencias de género explicitas: la construcción es realizada casi exclusivamente por los hombres, mientras que la limpieza y la preparación de alimentos son tareas de las mujeres («Una dama nunca va a alzar un adobe», hermano, 34 años). Esta división del trabajo puede ser flexible en caso las personas del sexo correspondiente a la labor no estén disponibles en el número adecuado.

En relación con el monitoreo al docente, los esfuerzos se centran en la puntualidad y asistencia, características claramente observables desde fuera del aula. Sin embargo, la vigilancia sobre lo que sucede una vez que el docente está dentro de su espacio de trabajo es un tema no explorado. Es posible que los padres y madres opten por no involucrarse en el trabajo pedagógico por respeto a la figura del docente (Hung, 2007), por desconocimiento de las formas y contenidos discutidos en el mismo (Gu, 2008; Balarin y Cueto, 2007; Benavides, 2007; Belaunde, 2006) o por temor a que reaccione de forma negativa ante esta «intrusión» en su disciplina: «A veces al reclamar, terminan por odiar a los alumnos» (madre, 38 años).

En lo que respecta a las percepciones, se ha observado que los padres tienden a considerar como indicador de logro académico la capacidad de lectoescritura de sus hijos. La maestría de esta habilidad parece ser el foco del refuerzo de los aprendizajes en casa también. Se considera que el énfasis en el desarrollo de esta capacidad puede deberse a múltiples causas. La primera es la facilidad de su comprobación; la revisión de cuadernos (también, una categoría usual de respuesta en la muestra) brinda luces acerca de la capacidad de los niños y niñas para escribir. La segunda es que los padres y madres pueden considerar que esta capacidad sirve de base para el desarrollo de habilidades futuras. El razonamiento de «Si no saben leer, no van a saber cómo aprender» también es compartido por el Gobierno: programas nacionales, como el Programa de Logros de Aprendizaje, y evaluaciones, como la Evaluación Censal 
de Estudiantes, se han enfocado en estas habilidades. Una tercera posibilidad, manifestada especialmente en declaraciones como "Para que sepan, aunque sea firmar su nombre» (madre, 40) da cuenta de la percepción de que la adquisición de esta habilidad es útil para la vida y que podría contribuir con la mejora del estatus. Esta última posibilidad podría ser especialmente importante para aquellos padres que cuentan con un nivel educativo básico.

Se visibiliza en los modos de involucramiento que la participación de las familias es necesaria para el funcionamiento regular, mantenimiento y crecimiento de la institución educativa. Los padres son la mano de obra que se encarga de la construcción de las aulas, cercos y servicios higiénicos, mientras que las madres se encargan de la limpieza y la preparación de los alimentos de los niños. De forma similar, se encuentra que la escuela también depende de financiamiento otorgado por los padres de familia. Antes de que los programas de mantenimiento de locales escolares o los concursos deportivos y florales del Minedu entren en vigencia, eran los fondos otorgados por los padres, ya sea a través de las cuotas de Apafa o mediante la participación de actividades profondos, los que se empleaban para financiar algunas actividades de las IE, tales como concursos de conocimiento, eventos deportivos y actividades cívicas, religiosas y culturales, así como la mejora de la infraestructura del local. Este modelo, presente en todos los centros visitados, manifiesta cómo la comunidad suple mediante su trabajo las falencias de los servicios prestados por el Estado.

A partir de ello, es pertinente en este acápite presentar la información concerniente a los espacios de participación «institucionalizados», como la Apafa y el Conei. En relación con la primera, se debe anotar que los padres de familia entrevistados manifiestan estar constantemente en contra de las decisiones de la Apafa; esta es muchas veces vista como ineficiente o una obligación que hay que cumplir para evitar la multa. Este hallazgo refuerza los resultados expuestos por Benavides et al. (2009). Es destacable como los padres no parecen considerarse dentro de la Apafa. Esta se percibe, más bien, como un organismo ajeno, manejado exclusivamente por la directiva que la encabeza, no por los padres que la integran. Fuera de la coordinación para los trabajos de mejora de infraestructura y para brindar el refrigerio de los niños, no se presentaron otras tareas específicas de esta asociación. La debilidad de este vínculo es especialmente perniciosa en tanto daña la «sensación de sociedad» identificada como necesaria para el trabajo organizado (Kyriakides, 2005). Por otro lado, a pesar de que los Conei están nominalmente presentes en todas las IE visitadas, ningún informante hizo referencia a este organismo. La presencia formal pero ausencia práctica de este consejo es coherente con el comportamiento de formas de participación impuestas desde arriba (Eguren, 2006).

Respecto a los modos de participación centrados en casa, encontramos que el apoyo en la realización de tareas escolares es uno de los modos de involucramiento de más alta preponderancia en la muestra. En las entrevistas con los padres, se encontraron dos conductas específicas dentro de esta cate88 I goría que es pertinente seguir explorando: varios de los participantes hacían 
diferencia entre la revisión de la tarea y el apoyo en la realización de la misma; la primera era una acción de vigilancia y corrección, mientras que la segunda implicaba más bien un trabajo conjunto. Se resalta que estas acciones no son mutuamente excluyentes, y se hipotetiza que la elección de uno u otro modo puede deberse a elementos estables, como diferencias en estilos de crianza (Lareau, 2002); a elementos contextuales, como la cantidad de tiempo disponible por los padres (Méndez, 2010; Kyriakides, 2005); el nivel de desarrollo del niño y su etapa escolar (Green, Walker, Hoover-Dempsey \& Sandler, 2007; Deslandes \& Bertrand, 2005); y el dominio del tema en cuestión (Hung, 2007; Bello \& Villarán, 2004).

Existen dos modos de involucramiento resaltantes que podrían considerarse específicos al medio rural. Mecanismos como «Enviar/matricular a sus hijos» (Mo11) o «Trabajar más para que su hijo no tenga que hacerlo» (Mo17) deben ser considerados teniendo en cuenta que la educación de los hijos puede ser un costo doble para los padres dedicados a labores agrarias. No implica solo el gasto en tiempo y dinero de los propios padres -descrito anteriormente-, sino que también involucra la pérdida de la mano de obra gratuita que sus hijos pueden brindar. Los padres, sin embargo, asumen estos costos bajo la creencia de que la educación es una inversión, no un gasto, el cual les permitirá salir adelante en el futuro. Dentro de este rubro, también se registra la participación en el programa Juntos (Mo14); este consiste -de manera resumida- en la entrega de un bono económico mensual para la familia, condicionado por la matrícula y asistencia de los estudiantes a la escuela (entre otras cosas). Este bono podría paliar los costos adicionales que el envío de los hijos al colegio podría involucrar en la familia.

\subsection{Factores asociados a la participación}

Al revisar los factores asociados a la participación, encontramos una distribución que tiende a centrarse en solo algunos de los posibles factores del modelo. Cada uno de estos puntos será desarrollado individualmente.

\subsubsection{Creencias motivacionales de los padres}

La construcción del rol parental es uno de los factores que presentan mayor variedad de conductas asociadas. Se considera que la participación de los padres y madres está vinculada a la valoración de la educación formal como un vehículo de mejora de estatus social. Los padres consideran que la educación es una escalera para que sus hijos puedan acceder a un trabajo profesional, fuera de la localidad y con ingresos estables. Un trabajo con estas características acarrea una mejora de estatus y calidad de vida futura (Ames, 2002).

A pesar de que los frutos de la labor parental son principalmente cosechados por los hijos, esto no implica que los padres (o la comunidad) no se beneficien. El tener hijos profesionales implica un logro propio de los padres. 
Encontramos que esto es ejemplificado en las palabras de una madre orgullosa: "Yo logré que dos (de mis hijos) sean profesores» (madre, 56). Es posible que el que sus hijos hayan alcanzado la profesionalización sea visto como el logro máximo de muchos padres, el cumplimiento óptimo de su rol paternal. A pesar de que la meta parece ser clara, las acciones específicas para lograrla no lo son. Estos resultados son similares a los reportados por Balarin y Cueto (2007).

La profesionalización de los hijos también acarrea otro beneficio reconocido por los padres, pues se espera que la inversión de tiempo y dinero que ha implicado la instrucción escolar retorne a la familia, especialmente, cuando los padres ya no estén en condiciones de seguir con su labor. Esto se manifiesta en declaraciones como: «(Que sean) Profesionales, que correspondan la inversión y esfuerzo» (padre, 35). Recordemos que, en este contexto, el cuidado y manutención de los adultos mayores es responsabilidad completa de sus hijos, pues no hay sistema alguno de pensiones reportado.

La búsqueda de la profesionalización de los hijos es un motivo bastante constructivo; sin embargo, encontramos que suele estar acompañado de una fuerte desvalorización del modo de vida actual. Esto se manifiesta en declaraciones como «De lo que hemos sido, ahí nos hemos quedado no más. Como agricultores, callamos» (padre, 40) o "Ya no sean como nosotros, ignorantes, brutos. Para que sean profesionales» (madre, 56). Estas citas evidencian la intensidad de este pensamiento y el posible impacto negativo que puede tener en la autoeficacia e identidad de los padres. Se esperaba, por estudios previos (Ames et al., 2009), que algunos docentes manifestaran opiniones negativas acerca de la forma de vida de los padres de familia de zona rural. A pesar de que efectivamente se registraron algunas, estas no alcanzaron, ni en intensidad ni en cantidad, las opiniones negativas dadas por los propios padres. Los efectos de una baja autoeficacia en materia educativa son particularmente graves por lo expuesto por Anderson y Minke (2007), quienes encontraron que una baja autoeficacia era la barrera más difícil de superar para incrementar los niveles de involucramiento parental. La situación de inactividad actual funciona de forma inversa al círculo virtuoso presentado por Hoover-Dempsey et al. (2001), a través del cual la autoeficacia se fortalecía mediante la participación exitosa. En este caso, encontramos que la ausencia de participación refuerza la baja autoeficacia sobre este aspecto específico, lo cual dificulta aún más una participación espontánea.

Producto de la revisión de las categorías creadas para este estudio, se ha incorporado una creencia motivacional adicional: "Aporte de los padres a la mejora de la comunidad». Los factores que se han incorporado en esta creencia motivacional están relacionados con un estilo de vida centrado en el grupo. Como ya se ha descrito anteriormente, las expectativas de éxito que tienen los padres son considerables, pero estas no circunscriben su influencia exclusivamente a la esfera privada. Encontramos que algunos esperan que sus hijos traigan desarrollo a la comunidad y sean fuente de orgullo de la familia: "(Que 90 I sean) Representantes de la comunidad fuera de la misma» (padre, 50). Este 
factor es bastante complejo en tanto implica el reconocimiento de los padres como «buenos padres», capaces de criar un hijo exitoso, que, además, genere beneficios para la comunidad en general.

El otro factor que se incluye en esta creencia es la búsqueda de mejoras en las condiciones físicas de la escuela. Ya se ha discutido anteriormente la importancia de un referente concreto visible que funge de indicador (un cuaderno lleno o un docente dentro del aula); en este caso, se busca una escuela «bonita», «segura», "grande» no solo para que los niños estudien, sino para que sirva de indicador del crecimiento y progreso de la comunidad.

\subsubsection{Percepciones de invitación a la participación}

En lo concerniente a las invitaciones por parte de la escuela, se encontró que se percibe que la única forma de garantizar la participación es a través de medidas extrínsecas, multas u otras formas de presión social: «Las invitaciones sin multa no funcionan, ya hemos intentado» (director, 39 años). Los profesores y directores entrevistados tampoco parecen mostrar mucha confianza en la voluntad o capacidad de los padres para afectar positivamente la formación escolar de sus hijos, especialmente, en temas abstractos: «Para que las reuniones tengan acogida no basta con que se traten sólo temas académicos. Se necesita hablar de infraestructura» (director-docente, 36). Estos resultados son similares a los encontrados por Benavides et al. (2009). Esta situación de desconfianza generalizada no aporta a la «sensación de sociedad», propuesta por Kyriakides (2005) como prerrequisito para el funcionamiento colaborativo de la escuela.

Resalta la ausencia de declaraciones sobre las invitaciones específicas del docente y de los hijos. Una hipótesis es que esto no se debe necesariamente a que ellos no produzcan invitaciones, sino que estas no son reconocidas como un factor determinante en la participación parental, sino solo una oportunidad para que los padres manifiesten o no la presencia de otros factores más intrínsecos.

Ahora, cabe anotar que un grupo de docentes mantiene algunas aprensiones sobre la inclusión de los padres en la esfera académica de la escuela. Esto puede responder a que se ha construido una división tácita de tareas entre la escuela y el hogar. La formación de los estudiantes debe darse en ambos espacios, pero el primero debe concentrarse en los aprendizajes, mientras en el segundo deben desarrollarse aspectos éticos y de comportamiento. Esta creencia puede generarse por dos razones, ya sea porque el hogar es el espacio donde deben desarrollarse los contenidos morales o porque en la casa no se cuentan con las capacidades necesarias para el desarrollo de contenidos escolares y la ética es lo único restante. 


\subsubsection{Contexto de vida percibido por los padres}

Durante las entrevistas, no se encontraron casos en los que se sostuviera que los padres no contaban con tiempo suficiente para involucrarse; este tema ni siquiera fue nombrado en las conversaciones mantenidas con los informantes. Es posible que, dadas las características de flexibilidad horaria de la labor agrícola en la zona, así como la cercanía geográfica de la escuela primaria a los hogares de las familias, el tiempo no sea un problema particular, como sí ha sucedido en estudios urbanos (Méndez, 2010; DePlanty, Coulter-Kern \& Duchane., 2007; Kyriakides, 2005). De forma similar, no se encontraron referencias a dificultades en la participación parental a causa de la presencia de un niño pequeño en la familia (McWayne et al., 2004). Esto se debe, casi con certeza, a que el cuidado de los niños en la zona recae exclusivamente en las madres. Las formas en las que ellas participan de la educación y el cuidado de un hijo pequeño no son excluyentes: las madres tienen la libertad de llevar a sus hijos pequeños a la escuela cuando se ocupan de la preparación de desayunos u otra actividad. Por otro lado, en casa, si es que colaboran en el desarrollo de las tareas, pueden ocuparse de revisarlas mientras los pequeños juegan o descansan.

Un aspecto que sí es detallado por los informantes está relacionado con la falta de conocimientos para el involucramiento. Tanto docentes como padres explicitan que, por su bajo nivel educativo alcanzado, el apoyo a sus hijos, especialmente en la esfera académica, es limitado. Estos resultados refuerzan los hallazgos de Bello y Villarán (2004), quienes proponen que la poca experiencia en el sistema educativo se ve acompañada de menor variabilidad de conductas de involucramiento.

\subsubsection{Aspectos adicionales}

Al iniciar la investigación se manejó como hipótesis que los hermanos mayores participarían en gran medida de la educación de sus hermanos menores, especialmente, en aquellos casos en los que los padres no tenían un nivel educativo elevado (Benavides et al., 2009). Se encuentra, sin embargo, que esta hipótesis no se cumple. Esto puede deberse a dos motivos. El primero es que los hermanos mayores no se encuentran en la zona. Las comunidades en las que se realizó el estudio no cuentan con escuelas de nivel secundario cercanas, por lo que los estudiantes de ese nivel tienen que trasladarse a otro pueblo para continuar su formación. La segunda posibilidad es que los hermanos mayores estén trabajando en otro lugar o estudiando sus propias lecciones. Revisando las progresiones de población elaboradas por el Instituto Nacional de Estadística e Informática (Inei, 2015) se encuentra un despoblamiento de 16\% de toda la provincia entre los años 2000 y 2015. Muchos informantes hicieron referencia al proyecto agroindustrial Chavimochic como uno de los causantes

92 I de este fenómeno. 
Otros agentes de la familia extendida (abuelos, tíos, primos, etc.) no se registraron en la muestra. Los informantes reportaron que las familias nucleares viven aisladas, lo cual se debe, en parte, a la forma de herencia de la tierra, que implica la partición del terreno entre los distintos hijos. Encontramos, entonces, que los únicos agentes de participación familiar son las madres y padres de los estudiantes.

\section{Conclusiones}

El presente estudio replica y sistematiza los resultados registrados en investigaciones previas con población similar (Ames et al., 2009; Benavides et al., 2006; Eguren, 2006; Gonzales, 2006 Montero, 2006; Saavedra \& Suarez, 2002). Se ha observado que, en general, que los padres y madres de familia aportan en gran medida al funcionamiento de la escuela, principalmente, brindando las condiciones físicas necesarias para que los estudiantes asistan a clases. Los padres tienen, también, en menor medida, cierta injerencia en la labor pedagógica, especialmente, apoyando en el desarrollo de tareas fuera del aula. Su apoyo en labores de gestión de la institución es, sin embargo, inexistente.

Al adaptar los resultados encontrados al modelo teórico de HooverDempsey y Sandler (1997), se encuentran algunos resultados interesantes. Los principales factores asociados a la decisión de involucrarse en la educación de los hijos están vinculados a la búsqueda de mejores condiciones de vida a través de una mayor capacidad migratoria y un mejor acceso a servicios de calidad. Esta condición de mejora beneficia a los niños y niñas en la escuela, pero también afecta a las familias y la comunidad de donde provienen. El lograr que los hijos sean profesionales parece ser el objetivo principal a cumplir en su rol como padres.

A pesar de que algunas formas de participación son intrínsecas a los padres, varios de ellos, especialmente en lo concerniente a la participación en la Apafa, reconocen que se involucran por obligación. De forma similar, en el discurso de los docentes, no se hace referencia a ningún tipo de invitación para la participación, sino que, más bien, parece centrarse en forzar a que los padres participen a través de distintas formas de presión.

Los diferentes actores entrevistados establecen como principal dificultad para un mayor involucramiento la ausencia de dominio de los contenidos escolares por parte de los padres. Los bajos niveles educativos alcanzados por ellos, así como una valoración negativa de su propia ocupación y estilo de vida, son reconocidos como una situación estable y tiñen sus declaraciones de cierta desesperanza. Es probable que cualquier intervención que busque incentivar la participación parental deba, primero, modificar esta creencia en toda la comunidad educativa.

Para este estudio, se ha incorporado un factor asociado adicional al modelo: «Aporte de los padres a la mejora de la comunidad». Esta es una creencia que busca resaltar la importancia de la comunidad como un elemento de la 
identidad de los padres. Ellos trabajan para la mejora de la misma a través de la crianza de hijos profesionales, así como en la construcción de infraestructura escolar segura y agradable. Ambas labores generan un indicador concreto de la eficacia de la comunidad. Este factor asociado es aún provisional y requiere mayor investigación para ser adecuadamente establecido.

Pese a estos hallazgos, el estudio presenta algunas debilidades. A pesar de haberse reconocido la importancia de las Apafa en los antecedentes, en esta investigación, no se tomó en cuenta la opinión de las personas que participaban en la directiva de estas asociaciones. Se sugeriría, para estudios posteriores, enriquecer el análisis incorporando intencionalmente a este grupo en la muestra. La categoría "Apoyar en la realización de tareas en casa» (Mo02) tiene manifestaciones conductuales muy variadas, que oscilan entre vigilar la realización del trabajo hasta realizar el trabajo con los hijos e hijas. Se considera necesario recoger información más precisa sobre este modo, sobre todo, dada su frecuencia en la muestra. Otro punto pendiente de exploración es la evaluación del nivel de interés por los padres y madres en involucrarse en la educación. Se espera que este interés varíe dependiendo tanto de los padres entrevistados como del modo particular de involucramiento planteado.

El presente estudio brinda algunas luces iniciales para investigaciones más precisas sobre los conceptos revisados. Para estudios posteriores, se sugiere profundizar en el análisis de los mismos según variables demográficas como el sexo y el nivel educativo (tanto de padres como de hijos), así como iniciar la vinculación de esta variable con otras de importancia, como los logros educativos de los estudiantes o la satisfacción con el servicio recibido.

\section{Nota biográfica}

\section{IgNACio Franco Vega}

Es bachiller en Psicología con mención en Psicología Social por la Pontificia Universidad Católica del Perú. Actualmente, trabaja como consultor e investigador de políticas y programas educativos. Sus temas de interés son la educación rural, y el diseño y monitoreo de programas sociales. 


\section{Referencias}

Ames, P. (2002). Educación en interculturalidad: repensando mitos, identidades y proyectos. En N. Fuller (Ed.), Interculturalidad y política. Lima: Red para el Desarrollo de las Ciencias Sociales en el Perú.

Ames, P., Rojas, V. \& Portugal, T. (2009). Empezando la escuela: ¿Quién está preparado? Investigando la transición al primer grado. Niños del Milenio (Documento de Trabajo 47). Lima: Grupo de Análisis para el Desarrollo (Grade).

Anderson, K. \& Minke, K. (2007). Parent involvement in education: Toward an understanding of parents' decision making. Journal of Educational Research, 100(5), 311-323.

Ansión, J. (1995). Del mito de la educación al proyecto educativo. En G. Portocarrero \& M. Valcárcel (Eds.), El Perú frente al siglo XXI. Lima: Fondo Editorial de la Pontificia Universidad Católica del Perú.

Balarin, M. \& Cueto, S. (2007). The quality of parental participation and student achievement in Peruvian government schools (Working Paper). Oxford: Young Lives.

Belaunde, C. (2006). Del currículo al aula: Reflexiones en torno a la incorporación de lo local en la escuela pública. En C. Montero (Ed.), Escuela y participación en el Perú: Temas y dilemas. Lima: Instituto de Estudios Peruanos.

Benavides, M. (2005). Las escuelas, las familias y el género. En P. Ames (Ed.), Las brechas invisibles: Desafíos para una equidad de género en la educación. Lima: Universidad Peruana Cayetano Heredia.

(2007). Lejos (aún) de la equidad: La persistencia de las desigualdades educativas en el Perú. En Grade (Ed.), Investigación, política $y$ desarrollo en el Perú. Lima: Grade.

Benavides, M; Olivera, I. \& Mena, M. (2006). De papás y mamás a hijos e hijas: Las aspiraciones sobre el futuro y rol de las familias en las actividades escolares en el Perú rural. En M. Benavides (Ed), Los Desafíos de la escolaridad en el Perú estudios sobre los procesos pedagógicos, los saberes previos y el rol de las familias. Lima: Grade.

Benavides, M., Rodrich, H. \& Mena, M. (2009). Niveles de acoplamiento y desacoplamiento en la relación familia - escuela en contextos rurales: El caso de una muestra de familias de Quispicanchis, Cusco. Revista Peruana de investigación educativa, 1(1), 7-30.-

Bello, M. \& Villarán, V. (2004). Educación, reformas y equidad en los países de los Andes y cono sur: Dos escenarios en el Perú. Buenos Aires: Unesco.

Carranza, F., You, S., Chhuon, V. \& Hudley, C. (2009). Mexican American adolescents' academic achievement and aspirations: The role of 
perceived parental educational involvement, acculturation, and selfesteem. Adolescence, 44(174).

Cheung, C. \& Pomerantz, E. (2012). Why does parents' involvement enhance children's achievement? The role of parent-oriented motivation. Journal of Educational Psychology, 104(3), 820-832.

Christenson, S., \& Sheridan, S. (2001). Schools and families: Creating essential connections for learning. New York, NY: Guilford Press

Cueto, S. (2004). Factores predictivos del rendimiento escolar, deserción e ingreso a educación secundaria en una muestra de estudiantes de zonas rurales del Perú. Education Policy Analysis Archives, 12(35), 1-41.

(2007). Las evaluaciones nacionales e internacionales de rendimiento escolar en el Perú: balance y perspectivas. En Grade (Ed.), Investigación, políticas y desarrollo en el Perú. Lima: Grade.

Deslandes, R. \& Bertrand, R. (2005). Motivation of parent involvement in secondary-level schooling. Journal of Educational Research, 98(3), 164175.

DePlanty, J., Coulter-Kern, R. \& Duchane, K. (2007). Perceptions of parent involvement in academic achievement. Journal of Educational Research, 100(6), 361-368.

Eccles, J. S. \& Harold, R. D. (1996). Family involvement in children's and adolescents' schooling. En A. Booth \& J. F. Dunn (Eds.), Family-school links: How do they affect educational outcomes (pp. 3-34). New York: Routledge.

Eguren, M. (2006). Enfoques y prácticas promovidos desde el estado, la sociedad civil y las escuelas. En C. Montero (Ed.), Escuela y participación en el Perú: Temas y dilemas. Lima: Instituto de Estudios Peruanos.

Emanique, J. \& Earl, J. (2009). Parental influence, school readiness and early academic achievement of African-American boys. The Journal of Negro Education, 78(3), p. 260-276.

Fan, X. \& Chen, M. (2001). Parental Involvement and Students' Academic Achievement: A Meta-Analysis. Educational Psychology Review, 13(1), 1-22.

Fantuzzo, J., Bulotsky-Shearer, R., McDermott, P., McWayne, C., Frye, D., \& Perlman, S. (2007). Investigation of dimensions of social-emotional classroom behavior and school readiness for low-income urban preschool children. School Psychology Review, 36(1), 44-62.

Fantuzzo, J., McWayne, C., Perry, M. \& Childs, S. (2004). Multiple dimensions of family involvement and their relations to behavioral and learning competencies for urban, low-income children. School Psychology Review, 33(4), 467-480.

Gonzales, N. (2006) Participación de los padres de familia en la educación: El casos de las asociaciones de padres de familia. En C. Montero (Ed.), 
Escuela y participación en el Perú: Temas y dilemas. Lima: Instituto de Estudios Peruanos.

Green, C., Walker, J., Hoover-Dempsey, K. \& Sandler, H. (2007). Parents' motivations for involvement in children's education: An empirical test of a theoretical model of parental involvement. Journal of Educational Psychology, 99(3), 532-544.

$\mathrm{Gu}$, W. (2008). New horizons and challenges in China's Public schools for parent involvement. Education, 128(4), 570-578.

Hill, N. \& Tyson, D. (2009). Parental involvement in middle school: A meta-analytic assessment of the strategies that promote achievement. Developmental Psychology, 45(3), 740-763.

Hoover-Dempsey, K. V., Battiato, A., Walker, J., Reed, R., DeJong, J. \& Jones, K. (2001). Parental Involvement in Homework. Educational Psychologist, 36(3), 195-209.

Hoover-Dempsey, K. V. \& Sandler, H. (1997). Why do parents become involved in their children's education? Review of Educational Research, 67(1), 3-42.

Hoover-Dempsey, K. V., Walker, J. M. T., \& Sandler, H. M., (2005). Parents' motivations for involvement in their children's education. En E. N. Patrikakou, R. P. Weisberg, S. Redding \& H. J. Walberg (Eds.), SchoolFamily Partnerships for Children's Success (pp. 40-56). New York, NY: Teachers College Press.

Hung, C. (2007). Family, schools and Taiwanese children's outcomes. Educational Research, 49(2), 115-125.

Instituto Nacional de Estadística e Informática (2015). Sistema de información: Población 2000 al 2015. Recuperado de http://proyectos.inei.gob.pe/web/ poblacion/\#

Kyriakides, L. (2005). Evaluating school policy on parents working with their children in class. Journal of Educational Research, 98(5), 281-298.

Lareau, A. (2002). Invisible Inequality: Social class and childrearing in black families and white families. American Sociological Review, 67(5), 747-776.

Ley General de Educación. Ley No 28044 (2003).

Martini, F. \& Senéchal, M. (2012). Learning literacy skills at home: Parent teaching, expectations, and child interest. Canadian Journal of Behavioural Science/Revue Canadienne des Sciences du Comportement, 44(3), 210-221.

McWayne, C., Hampton, V., Fantuzzo, J., Cohen, H. \& Sekino, Y. (2004). A multivariate examination of parent involvement and the social and academic competencies of urban kindergarten children. Psychology in the Schools, 41(3), 363-377.

Mendez, J. (2010). How can parents get involved in preschool? Barriers and engagement in education by ethnic minority parents of children attending Head Start. Cultural Diversity and Ethnic Minority Psychology, 16(1), 26-36. 
Millán, A. \& Lossio, F. (Eds.) (2005). Sumando esfuerzos: 10 experiencias de participación ciudadana en la gestión local. Lima: Red para el Desarrollo de las Ciencias Sociales.

Montero, C. (2006) Escuela y participación en el Perú. En C. Montero (Ed.), Escuela y participación en el Perú: Temas y dilemas. Lima: Instituto de Estudios Peruanos.

Murillo, J. (2007). Investigación iberoamericana sobre eficacia escolar. Bogotá: Convenio Andrés Bello.

Orealc/Unesco (2004) Participación de las familias en la educación infantil latinoamericana. Santiago de Chile: Editorial Trineo.

Saavedra, J. \& Suárez, P. (2002) El financiamiento de la educación pública en el Perú: El rol de las familias. Lima: Grade.

Walker, J. M., Wilkins, A. S., Dallaire, J., Sandler, H. M., \& Hoover-Dempsey, K. V. (2005). Parental involvement: Model revision through scale development. Elementary School Journal, 106(2), 85-104.

Yan, W., \& Lin, Q. (2005). Parent involvement and mathematics achievement: Contrast across racial and ethnic groups. Journal of Educational Research, 99(2), 116-127 